\title{
Error Rate Performance Analysis of Coded Free-Space Optical Links over Gamma-Gamma Atmospheric Turbulence Channels
}

\author{
Murat Uysal, Member, IEEE, Jing (Tiffany) Li, Member, IEEE, and Meng Yu, Student Member, IEEE
}

\begin{abstract}
Error control coding can be used over free-space optical (FSO) links to mitigate turbulence-induced fading. In this paper, we derive error performance bounds for coded FSO communication systems operating over atmospheric turbulence channels, considering the recently introduced gamma-gamma turbulence model. We derive a pairwise error probability (PEP) expression and then apply the transfer function technique in conjunction with the derived PEP to obtain upper bounds on the bit error rate. Simulation results are further demonstrated to confirm the analytical results.
\end{abstract}

Index Terms-Atmospheric turbulence channel, free-space optical communication, pairwise error probability, error performance analysis.

\section{INTRODUCTION}

W IRELESS optical communications, also known as free-space optical (FSO) communications, is a costeffective and high bandwidth access technique, which is receiving growing attention with recent commercialization successes [1]. With the potential high-data-rate capacity, low cost and particularly wide bandwidth on unregulated spectrum, FSO communications is an attractive solution for the "last mile" problem to bridge the gap between the end user and the fiber-optic infrastructure already in place. Its unique properties make it also appealing for a number of other applications, including metropolitan area network extensions, enterprise/local area network connectivity, fiber backup, back-haul for wireless cellular networks, redundant link and disaster recovery.

In FSO communications, optical transceivers communicate directly through the air to form point-to-point line-of-sight links. One severe impairment over FSO links is buildingsway as a result of wind loads, thermal expansion and weak earthquakes. The effect of building-sway on the FSO link reliability has been studied in detail [2], [3]. Another major impairment is the atmospheric turbulence, which occurs as a result of the variations in the refractive index due to

Manuscript received October 13, 2003; revised November 06, 2004; accepted June 16, 2005. The associate editor coordinating the review of this paper and approving it for publication was A. Boucouvalas. The work of M. Uysal is supported in part by an NSERC Special Research Opportunity grant SRO-PJ 305821-05 and in part through a gift from the National Institute of Standards and Technology (NIST). The work of J. Li is supported in part by the Commonwealth of Pennsylvania, Department of Community and Economic Development, through the Pennsylvania Infrastructure Technology Alliance (PITA), and in part by the Army Research Laboratory.

M. Uysal is with the Department of Electrical and Computer Engineering, University of Waterloo, Ontario, Canada, N2G3L1. (e-mail: muysal@ece.uwaterloo.ca).

J. Li and $\mathrm{M}$. Yu are with the Department of Electrical and Computer Engineering, Lehigh University, Bethlehem, PA, USA, 18015. (e-mail: \{jingli,mey3\}@ece.lehigh.edu)

Digital Object Identifier 10.1109/TWC.2006.03565. inhomogeneities in temperature and pressure fluctuations. The atmospheric turbulence results in fluctuations at the received signal, i.e. signal fading, also known as scintillation in optical communication terminology [4], severely degrading the link performance, particularly over link distances of $1 \mathrm{~km}$ or longer.

Error control coding as well as diversity techniques can be used over FSO links to improve the error rate performance [5]-[8] . In [7], [8], Zhu and Kahn studied the performance of coded FSO links assuming a log-normal channel model for atmospheric turbulence. Specifically, they derived an approximate upper bound on the pairwise error probability (PEP) for coded FSO links with intensity modulation/direct direction (IM/DD) and provided upper bounds on the bit error rate (BER) using the transfer function technique.

Although log-normal distribution is the most widely used model for the probability density function (pdf) of the irradiance due to its simplicity, this pdf model is only applicable to weak turbulence conditions [4]. As the strength of turbulence increases, multiple scattering effects must be taken into account. In such cases, log-normal statistics exhibit large deviations compared to experimental data. Furthermore, it has been observed that log-normal pdf underestimates the behavior in the tails as compared with measurement results. Since detection and fade probabilities are primarily based on the tails of the pdf, underestimating this region significantly affects the accuracy of performance analysis. Due to the limitations of log-normal model, many statistical models have been proposed over the years to describe atmospheric turbulence channels under a wide range of turbulence conditions, e.g. K distribution, I-K distribution, and log-normal Rician channel [4]. Error rate performance of coded FSO links assuming $\mathrm{K}$ distribution and I-K distribution have been already studied by the authors in [9].

In a recent series of papers on scintillation theory [10][12], Andrews et.al. introduced the modified Rytov theory and proposed gamma-gamma pdf as a tractable mathematical model for atmospheric turbulence. This model is a twoparameter distribution which is based on a doubly stochastic theory of scintillation and assumes that small-scale irradiance fluctuations are modulated by large-scale irradiance fluctuations of the propagating wave, both governed by independent gamma distributions. The gamma-gamma pdf can be directly related to atmospheric conditions and provides a good fit to experimental results. The performance analysis of an uncoded FSO link over the gamma-gamma turbulence channel can be already found in [4, p.235].

In this paper, we will investigate error rate performance of 
coded FSO links operating over atmospheric channels, where the turbulence-induced fading is described by the gammagamma distribution. The organization of the paper is as follows: In Section II, we review the gamma-gamma channel model under consideration. In Section III, an approximate PEP expression is derived for an FSO communication system with on-off keying (OOK) and in Section IV, we describe how BER performance can be obtained using transfer function technique in conjunction with the derived PEP expression. In section $\mathrm{V}$, we present numerical results to demonstrate the accuracy of the derived PEP in comparison to the exact PEP and also present bounds on the BER performance. Analytical results are further confirmed through Monte-Carlo simulations. Conclusions are presented in Section VI.

\section{Atmospheric Turbulence Channel Model}

There has been a significant research effort on finding an accurate and efficient channel model for atmospheric turbulence channels. Owing to its simplicity, log-normal distribution is the most widely used channel model, however its applicability is mainly restricted to weak turbulence conditions [4]. Alternative models where the irradiance fluctuation is modeled as the result of two multiplicative random processes include the Rician/Log-normal model [13], the Nakagami/Gamma model [14] and the Negative Exponential/Gamma model (also known as the K channel) [15] among others. Recently, Andrews et.al. proposed the modified Rytov theory [10]-[12], which defines the optical field as a function of perturbations which are due to large-scale and small-scale atmospheric effects. Specifically, the normalized irradiance is defined as the product of two random processes, i.e. $I=I_{x} I_{y}$, where $I_{x}$ and $I_{y}$ arise from large scale and small scale turbulent eddies and each of them follows gamma distribution. This leads to the so-called gamma-gamma pdf, i.e.

$$
f(I)=\frac{2(\alpha \beta)^{(\alpha+\beta) / 2}}{\Gamma(\alpha) \Gamma(\beta)} I^{\frac{\alpha+\beta}{2}-1} K_{\alpha-\beta}(2 \sqrt{\alpha \beta I}), I>0
$$

where $K_{a}(\cdot)$ is the modified Bessel function of the second kind of order a. Here, $\alpha$ and $\beta$ are the effective number of smallscale and large scale eddies of the scattering environment. Assuming spherical wave propagation, these parameters can be directly related to atmospheric conditions according to $[4$, p.237], [16] ${ }^{1}$

$$
\begin{aligned}
& \alpha=\left[\exp \left(\frac{0.49 \chi^{2}}{\left(1+0.18 d^{2}+0.56 \chi^{12 / 5}\right)^{7 / 6}}\right)-1\right]^{-1} \\
& \beta=\left[\exp \left(\frac{0.51 \chi^{2}\left(1+0.69 \chi^{12 / 5}\right)^{-5 / 6}}{\left(1+0.9 d^{2}+0.62 d^{2} \chi^{12 / 5}\right)^{5 / 6}}\right)-1\right]^{-1}
\end{aligned}
$$

where $\chi^{2}=0.5 C_{n}^{2} k^{7 / 6} L^{11 / 6}$ and $d=\left(k D^{2} / 4 L\right)^{1 / 2}$. Here, $k=2 \pi / \lambda$ is the optical wave number, $\lambda$ is the wavelength, $D$ is the diameter of the receiver collecting lens aperture and $L$ is the link distance in meters. $C_{n}^{2}$ stands for the index of refraction structure parameter and is altitude-dependent. Several $C_{n}^{2}$ profile models are available in the literature, but the

\footnotetext{
${ }^{1}$ There is a missing term in the formula for $\beta$ given by Eq. 94 of [4-Chapter 7]. The correct formula should be read as in our Eq. 3 [16]
}

most commonly used is the Hufnagle-Valley model described by [4]

$$
\begin{aligned}
C_{n}^{2}(h)= & 0.00594(v / 27)^{2}\left(10^{-5} h\right)^{10} \exp (h / 1000) \\
& +2.7 \times 10^{-6} \exp (-h / 1500)+A \exp (-h / 1000)
\end{aligned}
$$

where $h$ is the altitude in meters $(\mathrm{m}), v$ is the rms windspeed in meters per second $(\mathrm{m} / \mathrm{sec})$ and $A$ is a nominal value of $C_{n}^{2}(0)$ at the ground in $\mathrm{m}^{-2 / 3}$. For FSO links near the ground, $C_{n}^{2}$ can be taken approximately $1.7 \times 10^{-14} \mathrm{~m}^{-2 / 3}$ during daytime and $8.4 \times 10^{-15} \mathrm{~m}^{-2 / 3}$ at night. In general, $C_{n}^{2}$ varies from $10^{-13} \mathrm{~m}^{-2 / 3}$ for strong turbulence to $10^{-17}$ $\mathrm{m}^{-2 / 3}$ for weak turbulence with $10^{-15} \mathrm{~m}^{-2 / 3}$ often defined as a typical average value [17].

\section{DERIVATION OF PEP}

The PEP represents the probability of choosing the coded sequence $\hat{\boldsymbol{X}}=\left(\hat{x}_{1}, \hat{x}_{2}, \cdots, \hat{x}_{M}\right)$ when $\boldsymbol{X}=\left(x_{1}, x_{2}, \cdots, x_{M}\right)$ indeed was transmitted. We consider IM/DD links using on-off keying (OOK). Following [8], we assume that the noise can be modeled as additive white Gaussian noise (AWGN) with zero mean and variance $N_{0} / 2$, independent of the on/off state of the received bit. Under the assumption of perfect channel state information (CSI), the conditional PEP with respect to fading coefficients $\boldsymbol{I}=\left(I_{1}, I_{2}, \cdots, I_{M}\right)$ is given as [8].

$$
P(\boldsymbol{X}, \hat{\boldsymbol{X}} \mid \boldsymbol{I})=Q\left(\sqrt{\frac{\epsilon(\boldsymbol{X}, \hat{\boldsymbol{X}})}{2 N_{0}}}\right)
$$

where $Q(\cdot)$ is the Gaussian- $Q$ function and $\epsilon(\boldsymbol{X}, \hat{\boldsymbol{X}})$ is the energy difference between two codewords. Since OOK is used, the receiver would only receive signal light subjected to fading during on-state transmission. Thus, we have

$$
P(\boldsymbol{X}, \hat{\boldsymbol{X}} \mid \boldsymbol{I})=\left(\sqrt{\frac{E_{s}}{2 N_{0}} \sum_{k \in \Omega} I_{k}^{2}}\right)
$$

where $E_{s}$ is the total transmitted energy and $\Omega$ is the set of bit intervals' locations where $\boldsymbol{X}$ and $\hat{\boldsymbol{X}}$ differ from each other. Defining the signal-to-noise ratio as $\tau=E_{s} / N_{0}$ and using the alternative form for Gaussian- $Q$ function [18], i.e. $Q(x)=(1 / 2 \pi) \int_{0}^{\pi / 2} \exp \left(-x^{2} / \sin ^{2} \theta\right) \mathrm{d} \theta$, we obtain

$$
P(\boldsymbol{X}, \hat{\boldsymbol{X}} \mid \boldsymbol{I})=\frac{1}{\pi} \int_{0}^{\pi / 2} \prod_{k \in \Omega} \exp \left(-\frac{\tau}{4} \frac{I_{k}^{2}}{\sin ^{2} \theta}\right) \mathrm{d} \theta
$$

To obtain unconditional PEP, we need to take an expectation of (7) with respect to $I_{k}$. Under the assumption of perfect interleaving, we can exploit independency among fading coefficients $I_{k}$ and write

$$
\begin{aligned}
P(\boldsymbol{X}, \hat{\boldsymbol{X}}) & \left.=\frac{1}{\pi} \int_{0}^{\pi / 2} \prod_{k \in \Omega} \mathrm{E}_{I_{k}}\left[\exp \left(-\frac{\tau}{4} \frac{I_{k}^{2}}{\sin ^{2} \theta}\right)\right)\right] \mathrm{d} \theta \\
& =\frac{1}{\pi} \int_{0}^{\pi / 2}\left[\int_{0}^{\infty} \exp \left(-\frac{\tau}{4} \frac{I^{2}}{\sin ^{2} \theta}\right) f(I) \mathrm{d} I\right]^{|\Omega|} d \theta
\end{aligned}
$$

where $\mathrm{E}(\cdot)$ is the expectation operation and $|\Omega|$ is the cardinality of $\Omega$, which also corresponds to the length of error event. Here, $f(I)$ is the pdf for the gamma-gamma channel given by (1). A direct use of (1) in (8) yields an expression 


$$
\begin{gathered}
P(\boldsymbol{X}, \hat{\boldsymbol{X}})=\frac{1}{\pi} \int_{0}^{\pi / 2}\left[c_{1} \tau^{-\frac{\beta}{2}}(\sin \theta)^{\beta} \int_{0}^{\infty} \mu^{\alpha-\beta-1} \exp \left(-\alpha \mu-c_{2} \frac{\sqrt{2} \sin \theta}{\sqrt{\tau}} \mu^{-1}-O\left(\mu^{-2}\right)\right) d \mu\right]^{|\Omega|} d \theta \\
P(\boldsymbol{X}, \hat{\boldsymbol{X}}) \cong \frac{1}{\pi} \int_{0}^{\pi / 2}\left[2^{\frac{\alpha-\beta+4}{4}} c_{1}\left(\frac{c_{2}}{\alpha}\right)^{\frac{\alpha-\beta}{2}}\left(\frac{\sin \theta}{\sqrt{\tau}}\right)^{\frac{\alpha+\beta}{2}} K_{\alpha-\beta}\left(2^{5 / 4} \sqrt{\frac{c_{2} \alpha \sin \theta}{\sqrt{\tau}}}\right)\right]^{|\Omega|} d \theta
\end{gathered}
$$

which unfortunately does not have a closed form solution. To get around with this, we exploit the fact that the underlying distribution is a conditional gamma distribution with its mean $\mu$ following again a gamma distribution, and rewrite (8) as

$P(\boldsymbol{X}, \hat{\boldsymbol{X}})=\frac{1}{\pi} \int_{0}^{\pi / 2}\left\{\mathrm{E}_{\mu}\left\{\mathrm{E}_{I \mid \mu}\left[\exp \left(-\frac{\tau}{4} \frac{I^{2}}{\sin ^{2} \theta}\right)\right]\right\}\right\}^{|\Omega|} \mathrm{d} \theta$

For the gamma-gamma channel, the inner expectation in (9) gives

$$
\begin{aligned}
& \mathrm{E}_{I \mid \mu}\left[\exp \left(-\frac{\tau}{4} \frac{I^{2}}{\sin ^{2} \theta}\right)\right] \\
= & \frac{\beta^{\beta}}{\mu^{\beta} \Gamma(\beta)} \int_{0}^{\infty} I^{\beta-1} \exp \left(-\frac{\tau}{4} \frac{I^{2}}{\sin ^{2} \theta}-\frac{\beta I}{\mu}\right) \mathrm{d} I
\end{aligned}
$$

Using the result from [19, p. 382, Eq. 3.462.1], i.e.

$$
\begin{aligned}
& \int_{0}^{\infty} z^{v-1} \exp \left(-a z^{2}-b z\right) d z \\
= & (2 a)^{-v / 2} \Gamma(v) \exp \left(\frac{b^{2}}{8 a}\right) D_{-v}\left(\frac{b}{\sqrt{2 a}}\right)
\end{aligned}
$$

where $D_{p}(\cdot)$ is the parabolic cylinder function, we obtain

$$
\begin{array}{r}
\mathrm{E}_{I \mid \mu}\left[\exp \left(-\frac{\tau}{4} \frac{I^{2}}{\sin ^{2} \theta}\right)\right]=\frac{\beta^{\beta}}{\mu^{\beta}}\left(\frac{\tau}{2} \frac{1}{\sin ^{2} \theta}\right)^{-\beta / 2} \\
\cdot \exp \left(\frac{\beta^{2} \sin ^{2} \theta}{2 \mu^{2} \tau}\right) D_{-\beta}\left(\frac{\sqrt{2} \beta}{\mu \sqrt{\tau}} \sin \theta\right)
\end{array}
$$

Since the operation of expectation over $\mu$ required in (9) does not yield a closed form, we resort to the asymptotic expansion of the parabolic cylinder function given as [20, p.689, Eq.19.9]

$$
\begin{aligned}
D_{-\left(a+\frac{1}{2}\right)}(z)= & \frac{\sqrt{\pi}}{\Gamma\left(\frac{3}{4}+\frac{a}{2}\right)} 2^{-\left(\frac{a}{2}+\frac{1}{4}\right)} \\
& \cdot \exp \left(-\left(\sqrt{a}+\frac{1}{16} a^{-3 / 2}\right) z-O\left(z^{2}\right)\right)
\end{aligned}
$$

where $O\left(z^{n}\right)$ represents the terms with power equal or higher than $n$. The above holds for $z^{2} \ll a$ and this condition is easily satisfied in our case for high SNR values. Replacing the asymptotic expression in (12) and using the resulting expression in (9), we have (14) at the top of the page, where

$$
\begin{aligned}
& c_{1}=\frac{\sqrt{\pi} \alpha^{\alpha} \beta^{\beta}}{\Gamma(\alpha) \Gamma\left(\frac{\beta+1}{2}\right)} \\
& c_{2}=\beta\left(\sqrt{\beta-\frac{1}{2}}+\frac{1}{16}\left(\beta-\frac{1}{2}\right)^{-\frac{3}{2}}\right)
\end{aligned}
$$

Neglecting the higher order components in (14), the inner integral can be solved with the help of [19, p. 384, Eq. 3.471.9]

$$
\int_{0}^{\infty} z^{v-1} \exp \left(-a z-b \frac{1}{z}\right) \mathrm{d} z=2\left(\frac{b}{a}\right)^{v / 2} K_{v}(2 \sqrt{a b})
$$

where $a>0, b>0$. This yields the final form for PEP as (17) at the top of the page. It should be emphasized that (17) is an approximation since the higher order components in the asymptotic expansion of the parabolic cylinder function are neglected.

\section{BER PERFORMANCE}

PEP is the basic tool for the derivation of union bounds on the error rate performance of a coded communication system. A union bound on the average BER can be found as [21]

$$
P_{b} \leq \frac{1}{n} \sum_{\boldsymbol{X}} P(\boldsymbol{X}) \sum_{\boldsymbol{X} \neq \hat{\boldsymbol{X}}} q(\boldsymbol{X}, \hat{\boldsymbol{X}}) P(\boldsymbol{X}, \hat{\boldsymbol{X}})
$$

where $P(\boldsymbol{X})$ is the probability that the coded sequence $\boldsymbol{X}$ is transmitted, $q(\boldsymbol{X}, \hat{\boldsymbol{X}})$ is the number of information bit errors in choosing another coded sequence $\hat{\boldsymbol{X}}$ instead of $\boldsymbol{X}$ and $n$ is the number of information bits per transmission. Using transfer function bounding technique combined with the alternative form for the Gaussian- $Q$ function, an efficient method for the computation of (18) is given in $[18$, p. 510$]$ as

$$
P_{b} \leq \frac{1}{n} \sum_{\boldsymbol{X}} P(\boldsymbol{X}) \int_{0}^{\pi / 2}\left[\left.\frac{\partial}{\partial N} T(D(\theta), N)\right|_{N=1}\right] \mathrm{d} \theta,
$$

where $N$ is an indicator variable taking into account the number of bits in error. If we consider uniform error probability (UEP) codes, a symmetry property exists for this code family making the distance structure of a UEP code independent of the transmitted sequence [18]. This eliminates the need for averaging over all possible transmitted sequences. In this case, (19) simplifies to

$$
P_{b} \leq \frac{1}{x} \int_{0}^{\pi / 2}\left[\left.\frac{1}{n} \frac{\partial}{\partial N} T(D(\theta), N)\right|_{N=1}\right] \mathrm{d} \theta .
$$

In (19)-(20), $D(\theta)$ is defined based on the underlying PEP expression. In our case, using the integrand of PEP expression given by (17), we have

$$
\begin{array}{r}
D(\theta)=2^{\frac{\alpha-\beta+4}{4}} c_{1}\left(\frac{c_{2}}{\alpha}\right)^{\frac{\alpha-\beta}{2}}\left(\frac{\sin \theta}{\sqrt{\tau}}\right)^{\frac{\alpha+\beta}{2}} \\
\cdot K_{\alpha-\beta}\left(2^{5 / 4} \sqrt{\frac{c_{2} \alpha \sin \theta}{\sqrt{\tau}}}\right)
\end{array}
$$




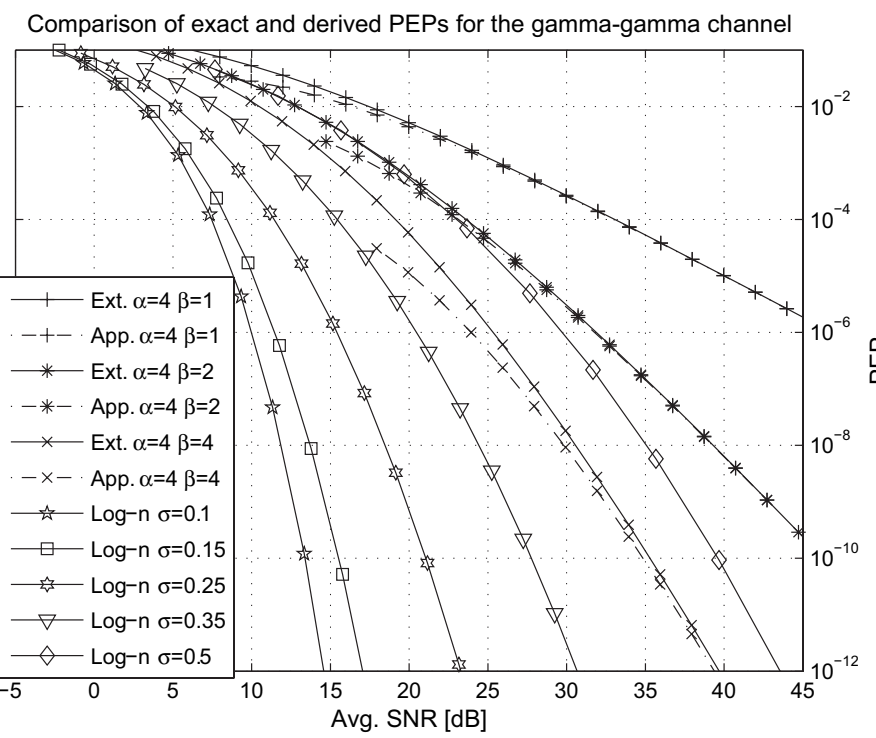

Fig. 1. Comparison of exact and derived PEPs for the gamma-gamma channel.

with $c_{1}$ and $c_{2}$ defined as in (15). In the case of exact PEP, $D(\theta)$ is given by

$$
\begin{gathered}
D(\theta)=\int_{0}^{\infty} \frac{2(\alpha \beta)}{\Gamma(\alpha) \Gamma(\beta)} I^{\frac{\alpha+\beta}{2}-1} \exp \left(-\frac{\tau}{4} \frac{I^{2}}{\sin ^{2} \theta}\right) \\
\cdot K_{\alpha-\beta}(2 \sqrt{\alpha \beta I}) d I
\end{gathered}
$$

\section{Numerical Results}

In this section, we will first compare the derived approximate PEP with the exact PEP expression. Then, as an example, we will consider a convolutionally coded FSO system and will use our PEP results to compute upper bounds on the BER of the considered system.

In Fig. 1, we plot the derived PEP approximation given by (17) for an error event of length 3 , i.e. $|\Omega|=3$, using different values of channel parameters $\alpha$ and $\beta$. We also compute the exact PEP given by (8) and include it as a reference (illustrated by dashed lines). It is observed from Fig.1 that the derived PEP provides a good approximation and coincides with the exact PEP for high signal-to-noise ratios. As a result of the neglected higher order terms in the approximation of parabolic cylinder function, the derived PEP behaves as a lower bound for the considered cases. In this figure, PEP curves for log-normal channels with typical standard deviation values of $\sigma=0.10,0.15,0.25,0.35,0.50^{2}$ have been further included. It is observed that these plots cover the (relatively) weak turbulence where error rate performance decays very fast with increasing SNR. On the other hand, our gamma-gamma results, with appropriate choices of $\alpha$ and $\beta$, can be used effectively for a wider range of turbulence conditions, including very strong turbulence. As revealed out by the numerical results under strong turbulence assumption, increasing SNR results in a relatively smaller change in the

\footnotetext{
${ }^{2}$ It should be noted that the PEP bounds presented in [8] for log-normal channels are not valid for $\sigma>0.25$ due to inefficiency of employed Taylor series approximation [8, p.1235]. Here, we consider exact PEP calculations for accuracy.
}

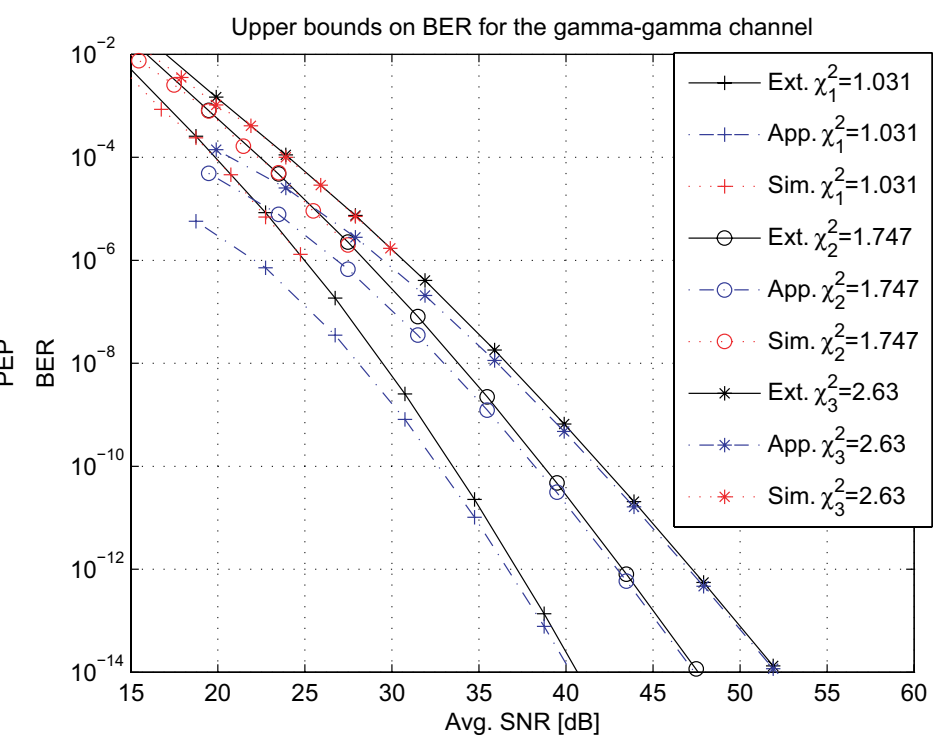

Fig. 2. Upper bounds on BER for the gamma-gamma channel.

slope of performance curves. This motivates the employment of powerful scintillation-mitigation techniques (in the form of coding and/or diversity) since it is not practical and even not feasible for many applications to increase the power margin in the link budget to eliminate the deep fades observed under strong turbulence.

In the following, we consider a convolutionally coded FSO communication system operating at $\lambda=1550 \mathrm{~nm}$. We assume $C_{n}^{2}=1.7 \times 10^{-14}$, which is a typical value of refractive index for FSO links near the ground during daytime. A point receiver is used, i.e. $D \ll L$ leading to $d=0$, therefore no aperture averaging is possible and system performance relies heavily on possible coding gains. In this case, $\alpha$ and $\beta$ are simply functions of $\chi$ as reflected by (2) and (3). We use a convolutional code, which has a code rate of $1 / 3$ and constraint length of 3 . The convolutional code under investigation is illustrated in Fig.8.2.2 of [21, p.471]. The transfer function of this code is found to be

$$
T(D(\theta), N)=\frac{D^{6}(\theta) N}{1-2 N D^{2}(\theta)} .
$$

Since the code satisfies the uniform error property, we can use (20) for BER performance evaluation, which leads to

$$
P_{b} \leq \frac{1}{\pi} \int_{0}^{\pi / 2} \frac{D^{6}(\theta) N}{\left(1-2 N D^{2}(\theta)\right)^{2}} \mathrm{~d} \theta
$$

where $D(\theta)$ is given by (21) and (22) for the derived approximate PEP bound and exact PEP, respectively. The average BER results are computed based on (24) in conjunction with (21) as well as with (22) to allow comparison with the true upper bound. Both of them are illustrated in the Fig. 2 for the FSO scenario under investigation. Here, we consider three different link distances $L_{1}=3000 \mathrm{~m}, L_{2}=4000 \mathrm{~m}$ and $L_{3}=5000 \mathrm{~m}$ which correspond to $\chi_{1}^{2}=1.031, \chi_{2}^{2}=1.747$ and $\chi_{3}^{2}=2.63$, respectively. For all three cases we considered, BER estimates based on the derived PEP yield a very good approximation to the true upper bound. Although there is some discrepancy in the lower SNR region, it provides excellent 
agreement as SNR increases. Monte-Carlo simulation results are furthermore included in Fig. 2 as a reference. Due to the long simulation time involved, we are able to give simulation results only up to $\mathrm{BER}=10^{-6}$. Simulation results are observed to be located slightly lower than the true upper bound and demonstrate an excellent agreement with the analytical results.

\section{CONCLUSION}

In this paper, we have investigated error rate performance of coded FSO systems operating over atmospheric turbulence channels, which are modeled with gamma-gamma distribution. Unlike the classically used log-normal assumption which is only accurate for modeling weak turbulence, the gammagamma channel model works for a variety of turbulence conditions. The parameters of this channel model are also easily related to practical system parameters such as the operating frequency, link distance, lens aperture giving valuable insights into FSO system performance. Considering this recently introduced channel model, we derived a PEP expression for coded FSO links with OOK. As a result of the underlying assumptions in the derivation, the derived PEP demonstrates some discrepancy in the lower SNR range, however coincides with the exact PEP for high SNRs. Adopting the transfer function technique associated with our PEP expression for the gamma-gamma channel, we have also obtained upper bounds on the BER performance, which have been further verified through Monte-Carlo simulations. Considering $\mathrm{BER}=10^{-9}$ is a practical performance target for an FSO link, our analytical results can serve as a simple and reliable method to estimate BER performance without resorting to lengthy simulations.

\section{ACKNOWLEDGEMENT}

The authors would like to thank L. C. Andrews (University of Central Florida) for bringing gamma-gamma channel model to their attention and C. N. Georghiades (Texas A\&M University) for helpful comments on an earlier draft version.

\section{REFERENCES}

[1] H. Willebrand and B. S. Ghuman, Free Space Optics: Enabling Optical Connectivity in Today's Networks. Sams Publishing, 2002.
[2] S. Arnon, "Optimization of urban optical wireless communication systems," IEEE Trans. Wireless Commun., vol. 2, no. 4, pp. 626-629, July 2003.

[3] S. Arnon, "Effects of atmospheric turbulence and building sway on optical wireless communication systems," Optics Letters, vol. 28, no. 2, pp. 129-131, Jan. 2003.

[4] L. C. Andrews, R. L. Phillips, and C. Y. Hopen, Laser Beam Scintillation with Applications, SPIE Press, 2001.

[5] F. M. Davidson and Y. T. Koh, "Interleaved convolutional coding for the turbulent atmospheric optical communication channel," IEEE Trans. Commun., vol.36, no. 9, pp. 993-1003, Sept. 1988.

[6] X. Zhu and J. M. Kahn, "Free-space optical communication through atmospheric turbulence channels," IEEE Trans. Commun., vol. 50, no. 8, pp. 1293-1300, Aug. 2002.

[7] X. Zhu and J. M. Kahn, "Pairwise codeword error probability for coded free-space optical communication through atmospheric turbulence channels," in Proc. IEEE ICC'01, pp. 161-164.

[8] X. Zhu and J. M. Kahn, "Performance bounds for coded free-space optical communications through atmospheric turbulence channels," IEEE Trans. Commun., vol. 51, no.8, pp. 1233-1239, Aug. 2003.

[9] M. Uysal and J. Li, "Error performance analysis of coded wireless optical links over atmospheric turbulence channels," in Proc. IEEE Wireless Communications and Networking Conf. (WCNC), vol. 4, pp. 2405-2410, Mar. 2004.

[10] L. C. Andrews, R. L. Phillips, C. Y. Hopen, and M. A. Al-Habash, "Theory of optical scintillation," Journal of Optical Society America A, vol. 16, no. 6, pp. 1417-1429, June 1999.

[11] L. C. Andrews, R. L. Phillips, and C. Y. Hopen, "Aperture averaging of optical scintillations: power fluctuations and the temporal spectrum," Waves Random Media, vol. 10, pp. 53-70, 2000.

[12] A. Al-Habash, L. C. Andrews, and R. L. Phillips, "Mathematical model for the irradiance probability density function of a laser beam propagating through turbulent media," Optical Engineering, vol. 40, no. 8, pp. 1554-1562, Aug. 2001.

[13] T. H. Churnside and S. F. Clifford, "Log-normal Rician probability density function of optical scintillations in the turbulent atmosphere," Journal of Optical Society America A, vol. 4, no. 10, pp. 1923-1930, Oct. 1987.

[14] L. C. Andrews and R. L. Phillips, "Mathematical genesis of the I-K distribution for random optical fields," Journal of Optical Society of America A, vol. 3, no. 11, pp. 1912-1919, Nov. 1986.

[15] E. Jakeman and P. N. Pusey, "The significance of K-distributions in scattering experiments," Physical Review Letters, vol. 40, no. 9, pp. 546-550, Feb. 1978.

[16] L. C. Andrews, private communication, Oct. 2004.

[17] J. W. Goodman, Statistical Optics, John Wiley \&Sons, 1985.

[18] M. K. Simon and M.-S. Alouini, Digital Communication over Fading Channels: A Uniform Approach to Performance Evaluation, New York: John Wiley\&Sons, 2000.

[19] I. S. Gradshteyn and I. M. Ryzhik, Table of Integrals, Series and Products, Academic Press, 1994.

[20] M. Abramowitz and I. S. Stegun, Handbook of Mathematical Functions with Formulas, Graphs and Mathematical Tables, Dover Publications, 1977.

[21] J. G. Proakis, Digital Communications, McGraw-Hill, 3rd ed., 1995. 